\title{
Message Handler
}

\author{
Dr. Steven Flank \\ Advanced Research Projects Agency \\ 3701 N. Fairfax Drive, Arlington, VA 22203 \\ Email: sflank@arpa.mil \\ Phone: (703) 696-2309
}

The Message Handler extracts critical intelligence information from the free-text of military messages to merge with the data parsed from fixed-fields. In the third of four project phases, the current focus is on developing stand-alone capabilities demonstrating the viability of natural language processing in Advance Concept Technology Demonstrations (ACTDs), and to operational users at military commands. E-Systems' engineers address the overall military message traffic processing problem, while SRI Interational's computational linguists adapt their Finite State Automaton Text Understanding System (FASTUS) technology to the domain of military message free-text.

In previous phases, the Message Handler processed over 180 types of messages while establishing the feasiblity of training the FASTUS technology to process the free-text of military messages. The previous efforts demonstrated that combining the FASTUS syntactical approach to processing freetext using finite-state automata with conventional parsing of fixed fields can yield results approximating human performance working under similar operational conditions. At the end of Phase II of the project, the Message Handler was able to process 4000 military messages per day. The automatically scored and manually validated results for messages without tables were $75 \%$ recall, $31 \%$ overgeneration, and $64 \%$ precision. The goals of the Message Handler project is to process 10,000 military messages per day at a performance level of 80 information extracted from the messages includes facilities, units, equipment, locations, times, coordinates, unit associations, events, event relationships, and parametric measures.

The message handling problem involves nuisances such as the structure and organization of both the overall message and the free-text. Another signif- icant message handling issue is the processing of unique ad-hoc tables which commonly appear within the free-text portion of messages. The Message Handler development reflects the evaluation of thousands of real world messages from the military theaters.

The voluminous and constantly changing flow of military messages necessitates an on-going review of the message corpus received by military commands to support the Message Handler development. In analyzing how the Message Handler functions match intelligence production environments, the system engineers established that the Message Handler should produce multiple interpretations of messages which precludes the denial of vital information to downstream systems that would correlate and fuse Message Handler outputs with data from other sources.

The Message Handler effort includes initiatives for making natural language processing a viable tool for the military user. Previous phases focused on developing extraction technology allowing minimal human intervention. The current phase assesses the appropriate level of human intervention and the appropriate interface tools. There is an on-going reassessment of what military-related language maintenance is amenable to execution by military users, and what maintenance must be reserved for linguistic specialists. The user tools will permit easy modification for lexicon or gazetteer and as much capability as possible for modifying domain-based grammars.

The Message Handler program has a collaborative engineering initiative between developer and potential users employing Internet and Intellink capabilities in the design and maintenance of the Message Handler. 